International Journal of Pure and Applied Mathematics

Volume 104 No. 3 2015, 389-406

ISSN: 1311-8080 (printed version); ISSN: 1314-3395 (on-line version)

url: http://www.ijpam.eu

doi: http://dx.doi.org/10.12732/ijpam.v104i3.9

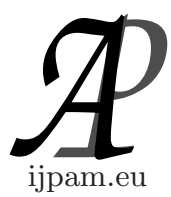

\title{
ULAM STABILITY OF A MIXED TYPE ADDITIVE-QUARTIC FUNCTIONAL EQUATION
}

\author{
K. Ravi ${ }^{1} \S$, S. Sabarinathan ${ }^{2}$ \\ ${ }^{1,2}$ Department of Mathematics \\ Sacred Heart College \\ Tirupattur, 635 601, TamilNadu, INDIA
}

Abstract: In this paper, we obtain the generalized Hyers-Ulam stability of a the functional equation

$$
\begin{aligned}
f(2 x+y)+f(x+2 y)-9 f(x+y)+f(x-y) & \\
& =f(2 x)-7 f(x)+8 f(-y)+f(y) .
\end{aligned}
$$

AMS Subject Classification: 39B82, 39B72

Key Words: additive functional equation, quartic functional equation, HyersUlam stability

\section{Introduction}

In 1940, S.M. Ulam [23] raised the following question concerning the stability of group homomorphisms:

"Let $G$ be a group and $H$ be a metric group with metric $d(.,$.$) . Given \epsilon>0$, does there exist a $\delta>0$ such that if a function $f: G \rightarrow H$ satisfies

$$
d(f(x y), f(x) f(y))<\delta
$$

for all $x, y \in G$, then there exists a homomorphism $a: G \rightarrow H$ with $d(f(x), a(x))$ $<\epsilon$ for all $x \in G$ ?"

Received: July 17, 2015

(C) 2015 Academic Publications, Ltd.

${ }_{\S}$ Correspondence author url: www.acadpubl.eu 
In 1941, D.H. Hyers [10] gave an answer to the Ulam's stability problem. He proved the following celebrated theorem.

Theorem 1. (D.H. Hyers) Let $X, Y$ be Banach spaces and let $f: X \rightarrow Y$ be a mapping satisfying

$$
\|f(x+y)-f(x)-f(y)\| \leq \epsilon
$$

for all $x, y \in X$. Then the limit

$$
a(x)=\lim _{n \rightarrow \infty} \frac{f\left(2^{n} x\right)}{2^{n}}
$$

exists for all $x \in X$ and $a: X \rightarrow Y$ is the unique additive mapping satisfying

$$
\|f(x)-a(x)\| \leq \epsilon
$$

for all $x \in X$.

In 1950, Aoki [2] generalized the Hyers theorem for additive mappings. In 1978, Th.M. Rassias [22] provided a generalized version of the theorem of Hyers which permitted the cauchy difference to become unbounded. Th.M. Rassias proved the following theorem for sum of powers of norms.

Theorem 2. (Th.M. Rassias) Let $X$ and $Y$ be two Banach spaces. Let $\theta \in[0, \infty)$ and let $p \in[0,1)$. If a function $f: X \rightarrow Y$ satisfies the inequality

$$
\|f(x+y)-f(x)-f(y)\| \leq \theta\left(\|x\|^{p}+\|y\|^{p}\right)
$$

for all $x, y \in X$. Then there exists a unique linear mapping $T: X \rightarrow Y$ such that

$$
\|f(x)-T(x)\| \leq \frac{2 \theta}{2-2^{p}}\|x\|^{p}
$$

for all $x \in X$. Moreover, if $f(t x)$ is continuous in $t$ for each fixed $x \in X$, then the function $T$ is linear.

The theorem of Th.M. Rassias was later extended for all $p \neq 1$. The stability phenomenon that was presented by Th.M. Rassias is called the generalized Hyers-Ulam stability.

Since then several stability problems for various functional equations have been investigated in (see [1], [3], [4], [9], [11], [12], [13], [15], [19], [20], [21]). Recently, the Ulam stability problem for mixed type additive-quadratic, additivecubic, additive-quartic, quadratic-cubic, quadratic-quartic, cubic-quartic, additive-quadratic-cubic, additive-cubic-quartic, quadratic-cubic-quartic and 
additive-quadratic-cubic-quartic functional equations have been considered in (see [6], [7], [8], [14], [16], [17], [18]).

In the year 2010, M.E. Gordji [8] obtained the general solution and generalized Hyers-Ulam stability of the mixed type quartic-additive functional equation

$$
\begin{aligned}
f(2 x+y)+ & f(2 x-y) \\
& =4[f(x+y)+f(x-y)]-\frac{3}{7}(f(2 y)-2 f(y))+2 f(2 x)-8 f(x) .
\end{aligned}
$$

In this paper, we consider the following functional equation deriving from additive and quartic functions

$$
\begin{aligned}
f(2 x+y)+f(x+2 y)-9 f(x+y)+ & f(x-y) \\
& =f(2 x)-7 f(x)+8 f(-y)+f(y) .
\end{aligned}
$$

It is easy to see that the function $f(x)=a x+b x^{4}$ is a solution of the functional equation (6). In this paper, we obtain the general solution and the generalized Hyers-Ulam stability of the functional equation (6).

\section{General Solution of Equation (6)}

In this section, we obtain the general solution of functional equation (6).

Theorem 3. Let $X, Y$ be vector spaces. An odd function $f: X \rightarrow Y$ satisfies (6) if and only if $f$ is additive.

Proof. Since $f$ is an odd function, equation (6) can be written as

$$
f(2 x+y)+f(x+2 y)-9 f(x+y)+f(x-y)=f(2 x)-7 f(x)-7 f(y)
$$

for all $x, y \in X$. Now, replacing $(x, y)$ by $(x, x)$ in $(7)$, we get

$$
f(3 x)=5 f(2 x)-7 f(x)
$$

for all $x \in X$. Now, plugging $(x, y)$ by $(0,2 x)$ in (7), we obtain

$$
f(4 x)=2 f(2 x)
$$

for all $x \in X$. Now, substituting $x$ by $\frac{x}{2}$ in (9), we have

$$
f(2 x)=2 f(x)
$$


for all $x \in X$. Now, setting $(x, y)=(x, x+2 y)$ in $(7)$ and using (10) in the resulting equation, we deduce that

$$
\begin{aligned}
2 f(x+2 y)=2 f(2 x & +y)+2 f(x-y) \\
& -18 f(x)+14 f(x+y)-10 f(y)
\end{aligned}
$$

for all $x, y \in X$. Replacing $(x, y)$ by $(x-y, x+y)$ in $(7)$, we get

$$
f(3 x+y)+f(3 x-y)=18 f(x)-7 f(x+y)-5 f(x-y)+2 f(y)
$$

for all $x, y \in X$. Switching $(x, y)$ to $(x+y, x-y)$ in (12), we obtain

$$
\begin{aligned}
2 f(2 x+y) & +2 f(x+2 y) \\
= & 18 f(x+y)-14 f(x)-10 f(y)+2 f(x-y)
\end{aligned}
$$

for all $x, y \in X$. By the virtue of equation (11) and after simplification, equation (13) can be written as

$$
f(2 x+y)=f(x+y)+f(x)
$$

for all $x, y \in X$. Now, replacing $(x, y)$ by $(x, y-x)$ in $(14)$, we obtain

$$
f(x+y)=f(x)+f(y)
$$

for all $x, y \in X$. This means that $f$ is an additive function.

Conversely, suppose $f$ is an additive function. Then $f$ satisfies (15). Replacing $y$ by $-y$ in (15), we get

$$
f(x-y)=f(x)-f(y)
$$

for all $x, y \in X$. Now, multiplying -9 in (15), we obtain

$$
-9 f(x+y)=-9 f(x)-9 f(y)
$$

for all $x, y \in X$. Summing the equation (16) with (17), we get

$$
f(x-y)-9 f(x+y)=-8 f(x)+8 f(-y)-2 f(y)
$$

for all $x, y \in X$. Plugging $x$ into $2 x$ in (15), we obtain

$$
f(2 x+y)=f(2 x)+f(y)
$$

for all $x, y \in X$. Now, switching $y$ to $2 y$ in (15), we get

$$
f(x+2 y)=f(x)+2 f(y)
$$

for all $x, y \in X$. Adding the equations (18), (19) and (20), we get equation (6). 
Theorem 4. Let $X, Y$ be vector spaces. An even function $f: X \rightarrow Y$ satisfies (6) if and only if $f$ is quartic.

Proof. Since $f$ is an even function, equation (6) can be written as

$$
\begin{aligned}
f(2 x+y) & +f(x+2 y) \\
& -9 f(x+y)+f(x-y)=f(2 x)-7 f(x)+9 f(y)
\end{aligned}
$$

for all $x, y \in X$. Plugging $(x, y)=(0,0)$ in $(21)$, we get $f(0)=0$. Now, replacing $(x, y)$ by $(0, x)$ in $(21)$, we obtain

$$
f(2 x)=16 f(x)
$$

for all $x \in X$. Now, subtituting $(x, y)=(x-y, x+y)$ in $(21)$, we get

$$
\begin{aligned}
f(3 x-y)+f & (3 x+y)-9 f(2 x)+f(2 y) \\
& =f(2 x-2 y)-7 f(x-y)+9 f(x+y)
\end{aligned}
$$

for all $x, y \in X$. Replacing $y$ by $-y$ in (23), we obtain

$$
\begin{aligned}
f(3 x+y)+f & (3 x-y)-9 f(2 x)+f(2 y) \\
& =f(2 x+2 y)-7 f(x+y)+9 f(x-y)
\end{aligned}
$$

for all $x, y \in X$. Setting $(x, y)=(x+y,-y)$ in $(21)$ and further simplification yields,

$$
\begin{gathered}
f(2 x+y)+f(x+2 y)=f(2 x+2 y)-7 f(x+y)+9 f(y) \\
+9 f(x)-f(x-y)
\end{gathered}
$$

for all $x, y \in X$. Using (21) in (37), we have

$$
f(2 x+2 y)=f(2 x)-16 f(x)+16 f(x+y)
$$

for all $x, y \in X$. Using (38) in (36), we obtain

$$
f(3 x+y)+f(3 x-y)=64 f(x)+9[f(x+y)+f(x-y)]-8 f(y)
$$

for all $x, y \in X$. Now, interchanging $x$ and $y$ and simplifying further, we get

$$
f(3 x)=81 f(x)
$$

for all $x \in X$. Using equations (22) and (28) in (39) and further simplification gives,

$$
f(3 x+y)+f(3 x-y)=9[f(x+y)+f(x-y)+2[f(3 x)-9 f(x)]
$$


for all $x, y \in X$, which is a quartic functional equation dealt by M.E. Gordji in his paper [5] with $n=3$.

Conversely, suppose $f$ is quartic. Then $f$ satisfies the standard quartic functional equation of the form

$$
\begin{aligned}
f(n x+y) & +f(n x-y) \\
=n^{2}[f(x+y)+f(x-y)]+2\left[f(n x)-n^{2} f(x)\right] & \\
& -2\left(n^{2}-1\right) f(y)
\end{aligned}
$$

for fixed integer $n \neq 0, \pm 1$, for all $x, y \in X$. Now, when $n=2$, the equation (30) becomes

$$
f(2 x+y)+f(2 x-y)=4[f(x+y)+f(x-y)]+24 f(x)-6 f(y)
$$

for all $x, y \in X$. Replacing $(x, y)$ by $\left(\frac{2 x-y}{2}, y\right)$ in (31) and simplifying further, we get

$$
\begin{aligned}
& 64 f(x)+64 f(x-y) \\
& \quad=f(2 x+y)+f(2 x-3 y)+6 f(2 x-y)-24 f(y)
\end{aligned}
$$

for all $x, y \in X$. Plugging $(x, y)$ into $(x-y, y)$ in $(31)$, we obtain

$$
f(2 x-3 y)=4 f(x)+4 f(x-2 y)+24 f(x-y)-6 f(y)-f(2 x-y)
$$

for all $x, y \in X$. Using (33) in (32), we arrive at

$$
\begin{aligned}
5 f(2 x-y)+f(2 x & +y)+4 f(x-2 y) \\
& =60 f(x)+40 f(x-y)+30 f(y)
\end{aligned}
$$

for all $x, y \in X$. Now, replacing $y$ by $-y$ in (34) and simplifying further, we get

$$
\begin{aligned}
4[f(2 x+y)+f(x+2 y)] & +f(2 x+y)+f(2 x-y) \\
= & 60 f(x)+40 f(x+y)+30 f(y)
\end{aligned}
$$

for all $x, y \in X$. Using (31) in (35) and using $f$ is quartic, we arrive at (6). This completes the proof of the theorem.

Theorem 5. Let $X, Y$ be vector spaces, and let $f: X \rightarrow Y$ be a function. Then $f$ satisfies (1) if and only if there exists a unique additive function $A: X \rightarrow$ $Y$ and a unique symmetric multi-additive function $B: X^{4} \times X \times X \times X \rightarrow Y$ such that $f(x)=A(x)+B(x, x, x, x)$ for all $x \in X$. 
Proof. Define the mappings $A, Q: X \rightarrow Y$ by

$$
A(x)=\frac{f(x)-f(-x)}{2}, \quad Q(x)=\frac{f(x)+f(-x)}{2}
$$

for all $x \in X$. Then we have $A(0)=0$ and $A(-x)=-A(x), Q(-x)=Q(x)$, for all $x \in X$. Also

$$
\begin{array}{r}
A(2 x+y)+A(x+2 y)-9 A(x+y)+A(x-y) \\
=A(2 x)-7 A(x)+8 A(-y)+A(y)
\end{array}
$$

and

$$
\begin{array}{r}
Q(2 x+y)+Q(x+2 y)-9 Q(x+y)+Q(x-y) \\
=Q(2 x)-7 Q(x)+8 Q(-y)+Q(y)
\end{array}
$$

for all $x, y \in X$. Now, substituting $x=0$ and $y=x$ in (36), we get

$$
A(2 x)=2 A(x)
$$

for all $x \in X$. Now, putting $x=0$ and $y=x$ in (37), we obtain

$$
Q(2 x)=16 Q(x)
$$

for all $x \in X$. Substituting (38) in (36), we get

$$
A(2 x+y)+A(x+2 y)-9 A(x+y)+A(x-y)=-5 A(x)-7 A(y)
$$

for all $x \in X$. Theorem 3 guarantees that $A$ is additive.

Using (39) in (37), we obtain

$$
Q(2 x+y)+Q(x+2 y)-9 Q(x+y)+Q(x-y)=9 Q(x)+9 Q(y)
$$

for all $x \in X$. Theorem 4 guarantees that $Q$ is quartic. Hence there exists a unique multi-additive function $B: X \times X \times X \times X \rightarrow Y$ and a unique additive function $A: X \rightarrow Y$ such that

$$
f(x)=A(x)+B(x, x, x, x)
$$

for all $x \in X$.

Conversely, suppose if $f(x)=A(x)+B(x, x, x, x)$, then we have $A(2 x)=$ $2 A(x)$ and $Q(2 x)=16 Q(x)$, for all $x \in X$. Hence it is easy to show that $f$ satisfies (1), which completes the proof of the theorem. 


\section{Generalized Hyers-Ulam Stability of Equation (6)}

Throughout this section, $X$ and $Y$ will be a real normed space and a real Banach space, respectively. Let $f: X \rightarrow Y$ be a function. Then we define $D_{f}: X \times X \rightarrow Y$ by

$$
\begin{array}{r}
D_{f} f(x, y)=f(2 x+y)+f(x+2 y)-9 f(x+y)+f(x-y) \\
-f(2 x)-7 f(x)+8 f(-y)+f(y)
\end{array}
$$

for all $x, y \in X$. Let $N$ denote the set of natural numbers.

Theorem 6. Let $\phi: X \times X \rightarrow[0, \infty)$ be a function satisfies

$$
\sum_{i=0}^{\infty} \frac{1}{2^{i}} \phi\left(0,2^{i} x\right)<\infty
$$

for all $x \in X$ with the condition

$$
\lim _{n \rightarrow \infty} \frac{\phi\left(2^{n} x, 2^{n} y\right)}{2^{n}}=0
$$

for all $x, y \in X$. If $f: X \rightarrow Y$ is an odd function such that $f(0)=0$, and that

$$
\left\|D_{f}(x, y)\right\| \leq \phi(x, y)
$$

for all $x, y \in X$, then there exists a unique additive function $A: X \rightarrow Y$ satisfying (1) and

$$
\|f(x)-A(x)\| \leq \frac{1}{2} \sum_{i=0}^{\infty} \frac{1}{2^{i}} \phi\left(0,2^{i} x\right)
$$

for all $x \in X$.

Proof. Replacing $(x, y)$ by $(0, x)$ in $(40)$ and dividing by 2 , we get

$$
\left\|\frac{1}{2} f(2 x)-f(x)\right\| \leq \frac{1}{2} \phi(0, x)
$$

for all $x \in X$. Now, plugging $x$ by $2 x$ in (41), dividing by 2 and then adding the resulting inequality with $(41)$, we obtain

$$
\left\|\frac{1}{2^{2}} f\left(2^{2} x\right)-f(x)\right\| \leq \frac{1}{2} \sum_{i=0}^{1} \frac{1}{2^{i}} \phi\left(0,2^{i} x\right)
$$


for all $x \in X$. Proceeding further and using induction on a positive integer $n$, we get

$$
\left\|\frac{1}{2^{n}} f\left(2^{n} x\right)-f(x)\right\| \leq \frac{1}{2} \sum_{i=0}^{n-1} \frac{1}{2^{i}} \phi\left(0,2^{i} x\right)
$$

for all $x \in X$. Now, replacing $x$ by $2^{m} x$ in (43) and dividing by $2^{m}$, we obtain

$$
\begin{aligned}
\left\|\frac{f\left(2^{m+n} x\right)}{2^{m+n}}-\frac{f\left(2^{m} x\right)}{2^{m}}\right\| & =\frac{1}{2^{m}}\left\|\frac{1}{2^{n}} f\left(2^{n} 2^{m} x\right)-f\left(2^{m} x\right)\right\| \\
& \leq \frac{1}{2^{m+1}} \sum_{i=0}^{n-1} \frac{1}{2^{i}} \phi\left(0,2^{m+i} x\right) \\
& \leq \frac{1}{2} \sum_{i=0}^{\infty} \frac{1}{2^{m+i}} \phi\left(0,2^{m+i} x\right)
\end{aligned}
$$

for all $x \in X$. This shows that $\left\{\frac{f\left(2^{\mathrm{n}} x\right)}{2^{\mathrm{n}}}\right\}$ is a Cauchy sequence in $Y$, as $m \rightarrow \infty$. Since $Y$ is a Banach space, then the sequence $\left\{\frac{f\left(2^{\mathrm{n}} x\right)}{2^{\mathrm{n}}}\right\}$ converges. We define $A: X \rightarrow Y$ by $A(x)=\lim _{n \rightarrow \infty} \frac{f\left(2^{n} x\right)}{2^{n}}$ for all $x \in X$. Since $f$ is odd function, then $A$ is odd. On the other hand, we have

$$
\begin{aligned}
\left\|D_{A}(x, y)\right\| & =\lim _{n \rightarrow \infty} \frac{1}{2^{n}}\left\|D_{f}\left(2^{n} x, 2^{n} y\right)\right\| \\
& \leq \lim _{n \rightarrow \infty} \frac{\phi\left(2^{n} x, 2^{n} y\right)}{2^{n}}=0
\end{aligned}
$$

for all $x, y \in X$. Hence by Theorem 3, $\mathrm{A}$ is an additive function. To show that $A$ is unique, suppose that there exists another additive function $A^{\prime}: X \rightarrow Y$ which satisfies (1) and (41). We have $A\left(2^{n} x\right)=2^{n} A(x)$ and $A^{\prime}\left(2^{n} x\right)=2^{n} A^{\prime}(x)$, for all $x \in X$. It follows that

$$
\begin{aligned}
\left\|A^{\prime}(x)-A(x)\right\| & =\frac{1}{2^{n}}\left\|A^{\prime}\left(2^{n} x\right)-A\left(2^{n} x\right)\right\| \\
& \leq \frac{1}{2^{n}}\left(\left\|A^{\prime}\left(2^{n} x\right)-f\left(2^{n} x\right)\right\|+\left\|f\left(2^{n} x\right)-A\left(2^{n} x\right)\right\|\right) \\
& \leq \sum_{i=0}^{\infty} \frac{\phi\left(0,2^{n+i} x\right)}{2^{n+i}}
\end{aligned}
$$

for all $x \in X$. By taking $n \rightarrow \infty$ in this inequality, we have $A^{\prime}(x)=A(x)$. 
Theorem 7. Let $\phi: X \times X \rightarrow[0, \infty)$ be a function satisfies

$$
\sum_{i=0}^{\infty} 2^{i} \phi\left(0,2^{-i-1} x\right)<\infty
$$

for all $x \in X$ with the condition

$$
\lim _{n \rightarrow \infty} 2^{n} \phi\left(2^{-n} x, 2^{-n} y\right)=0
$$

for all $x, y \in X$. Suppose that an odd function $f: X \rightarrow Y$ satisfies $f(0)=0$, and (40) for all $x, y \in X$, then there exists a unique additive function $A: X \rightarrow Y$ satisfying (1) and

$$
\|f(x)-A(x)\| \leq \sum_{i=0}^{\infty} 2^{i} \phi\left(0,2^{-i-1} x\right)
$$

for all $x \in X$.

Proof. Replacing $x$ by $\frac{x}{2}$ in (42) and multiplying by 2, we get

$$
\left\|f(x)-2 f\left(\frac{x}{2}\right)\right\| \leq \phi\left(0,2^{-1} x\right)
$$

for all $x \in X$. Again replacing $x$ by $\frac{x}{2}$ in (45) and summing the resulting inequality with (45), we get

$$
\left\|f(x)-2^{2} f\left(\frac{x}{2^{2}}\right)\right\| \leq \sum_{i=0}^{1} 2^{i} \phi\left(0,2^{-i-1} x\right)
$$

for all $x \in X$. By induction on $n \in N$, we have

$$
\left\|f(x)-2^{n} f\left(\frac{x}{2^{n}}\right)\right\| \leq \sum_{i=0}^{n-1} 2^{i} \phi\left(0,2^{-i-1} x\right)
$$

for all $x \in X$. Replacing $x$ by $2^{-m} x$ in (46) and multiplying by $2^{m}$, we obtain

$$
\begin{aligned}
\left\|2^{m+n} f\left(2^{-m-n} x\right)-2^{m} f\left(2^{-m} x\right)\right\| & =2^{m}\left\|f\left(2^{-n} 2^{-m} x\right)-f\left(2^{-m} x\right)\right\| \\
& \leq 2^{m} \sum_{i=0}^{n-1} 2^{i} \phi\left(0,2^{-i-1} x\right) \\
& \leq \sum_{i=0}^{\infty} 2^{m+i} \phi\left(0,2^{-i-1} x\right)
\end{aligned}
$$


for all $x \in X$. It follows that $\left\{2^{n} f\left(2^{-n} x\right)\right\}$ is a Cauchy sequence in $Y$ as $m \rightarrow \infty$. Since $Y$ is a Banach space, the sequence $\left\{2^{n} f\left(2^{-n} x\right)\right\}$ converges. Now, we define $A: X \rightarrow Y$ by $A(x)=\lim _{n \rightarrow \infty} 2^{n} f\left(2^{-n} x\right)$, for all $x \in X$. The rest of proof is similar to that of Theorem 6 .

Theorem 8. Let $\phi: X \times X \rightarrow[0, \infty)$ be a function satisfies

$$
\sum_{i=0}^{\infty} \frac{1}{16^{i}} \phi\left(0,2^{i} x\right)<\infty
$$

for all $x \in X$ with the condition

$$
\lim _{n \rightarrow \infty} \frac{\phi\left(2^{n} x, 2^{n} y\right)}{16^{n}}=0
$$

for all $x, y \in X$. If $f: X \rightarrow Y$ is an even function such that $f(0)=0$, and that

$$
\left\|D_{f}(x, y)\right\| \leq \phi(x, y)
$$

for all $x, y \in X$, then there exists a unique additive function $Q: X \rightarrow Y$ satisfying (1) and

$$
\|f(x)-Q(x)\| \leq \frac{1}{16} \sum_{i=0}^{\infty} \frac{1}{16^{i}} \phi\left(0,2^{i} x\right)
$$

for all $x \in X$.

Proof. Replacing $(x, y)$ by $(0, x)$ in $(47)$ and dividing by 16 , we get

$$
\left\|\frac{1}{16} f(2 x)-f(x)\right\| \leq \frac{1}{16} \phi(0, x)
$$

for all $x \in X$. Now, substituting $x$ by $2 x$ in (49), dividing by 16 and then adding the resulting inequality with (49), we obtain

$$
\left\|\frac{1}{16^{2}} f\left(2^{2} x\right)-f(x)\right\| \leq \frac{1}{16} \sum_{i=0}^{1} \frac{1}{16^{i}} \phi\left(0,2^{i} x\right)
$$

for all $x \in X$. Proceeding further and using induction on a positive integer $n$, we get

$$
\left\|\frac{1}{16^{n}} f\left(2^{n} x\right)-f(x)\right\| \leq \frac{1}{16} \sum_{i=0}^{n-1} \frac{1}{16^{i}} \phi\left(0,2^{i} x\right)
$$


for all $x \in X$. Now, replacing $x$ by $2^{m} x$ in (50) and dividing by $16^{m}$, we obtain

$$
\begin{aligned}
\left\|\frac{f\left(16^{m+n} x\right)}{2^{m+n}}-\frac{f\left(2^{m} x\right)}{16^{m}}\right\| & =\frac{1}{16^{m}}\left\|\frac{1}{2^{n}} f\left(2^{n} 2^{m} x\right)-f\left(2^{m} x\right)\right\| \\
& \leq \frac{1}{16^{m+1}} \sum_{i=0}^{n-1} \frac{1}{16^{i}} \phi\left(0,2^{m+i} x\right) \\
& \leq \frac{1}{16} \sum_{i=0}^{\infty} \frac{1}{16^{m+i}} \phi\left(0,2^{m+i} x\right)
\end{aligned}
$$

for all $x \in X$. This shows that $\left\{\frac{f\left(2^{\mathrm{n}} x\right)}{16^{\mathrm{n}}}\right\}$ is a Cauchy sequence in $Y$, as $m \rightarrow \infty$. Since $Y$ is a Banach space, then the sequence $\left\{\frac{f\left(2^{\mathrm{n}} x\right)}{16^{\mathrm{n}}}\right\}$ converges. We define $Q: X \rightarrow Y$ by $Q(x)=\lim _{n \rightarrow \infty} \frac{f\left(2^{n} x\right)}{16^{n}}$ for all $x \in X$. Since $f$ is even function, then $Q$ is even. On the other hand, we have

$$
\begin{aligned}
\left\|D_{Q}(x, y)\right\| & =\lim _{n \rightarrow \infty} \frac{1}{16^{n}}\left\|D_{f}\left(2^{n} x, 2^{n} y\right)\right\| \\
& \leq \lim _{n \rightarrow \infty} \frac{\phi\left(2^{n} x, 2^{n} y\right)}{16^{n}}=0
\end{aligned}
$$

for all $x, y \in X$. Hence by Theorem 3, $\mathrm{Q}$ is a quartic function. To show that $Q$ is unique, suppose that there exists another additive function $Q^{\prime}: X \rightarrow Y$ which satisfies (1) and (47). We have $Q\left(2^{n} x\right)=16^{n} Q(x)$ and $Q^{\prime}\left(2^{n} x\right)=16^{n} Q^{\prime}(x)$, for all $x \in X$. It follows that

$$
\begin{aligned}
\left\|Q^{\prime}(x)-Q(x)\right\| & =\frac{1}{16^{n}}\left\|Q^{\prime}\left(2^{n} x\right)-Q\left(2^{n} x\right)\right\| \\
& \leq \frac{1}{16^{n}}\left(\left\|Q^{\prime}\left(2^{n} x\right)-f\left(2^{n} x\right)\right\|+\left\|f\left(2^{n} x\right)-Q\left(2^{n} x\right)\right\|\right) \\
& \leq \frac{1}{8} \sum_{i=0}^{\infty} \frac{\phi\left(0,2^{n+i} x\right)}{16^{n+i}}
\end{aligned}
$$

for all $x \in X$. By taking $n \rightarrow \infty$ in this inequality, we have $Q^{\prime}(x)=Q(x)$.

Theorem 9. Let $\phi: X \times X \rightarrow[0, \infty)$ be a function satisfies

$$
\sum_{i=0}^{\infty} 16^{i} \phi\left(0,2^{-i-1} x\right)<\infty
$$

for all $x \in X$ with the condition

$$
\lim _{n \rightarrow \infty} 16^{n} \phi\left(2^{-n} x, 2^{-n} y\right)=0
$$


for all $x, y \in X$. Suppose that an even function $f: X \rightarrow Y$ satisfies $f(0)=0$, and (47) for all $x, y \in X$, then there exists a unique quartic function $Q: X \rightarrow Y$ satisfying (1) and

$$
\|f(x)-Q(x)\| \leq \sum_{i=0}^{\infty} 16^{i} \phi\left(0,2^{-i-1} x\right)
$$

for all $x \in X$.

Proof. Replacing $x$ by $\frac{x}{2}$ in (47), we get

$$
\left\|f(x)-16 f\left(\frac{x}{2}\right)\right\| \leq \phi\left(0,2^{-1} x\right)
$$

for all $x \in X$. Again replacing $x$ by $\frac{x}{2}$ in (52) and summing the resulting inequality with (52), we get

$$
\left\|f(x)-16^{2} f\left(\frac{x}{2^{2}}\right)\right\| \leq \sum_{i=0}^{1} 16^{i} \phi\left(0,2^{-i-1} x\right)
$$

for all $x \in X$. By induction on $n \in N$, we have

$$
\left\|f(x)-16^{n} f\left(\frac{x}{2^{n}}\right)\right\| \leq \sum_{i=0}^{n-1} 16^{i} \phi\left(0,2^{-i-1} x\right)
$$

for all $x \in X$. Replacing $x$ by $2^{-m} x$ in (46) and multiplying by $16^{m}$, we obtain

$$
\begin{aligned}
\| 16^{m+n} f\left(2^{-m-n} x\right)- & 16^{m} f\left(2^{-m} x\right) \| \\
& =16^{m}\left\|f\left(2^{-n} 2^{-m} x\right)-f\left(2^{-m} x\right)\right\| \\
\leq & 16^{m} \sum_{i=0}^{n-1} 2^{i} \phi\left(0,2^{-i-1} x\right) \\
\leq & \sum_{i=0}^{\infty} 16^{m+i} \phi\left(0,2^{-i-1} x\right)
\end{aligned}
$$

for all $x \in X$. It follows that $\left\{16^{n} f\left(2^{-n} x\right)\right\}$ is a Cauchy sequence in $Y$ as $m \rightarrow \infty$. Since $Y$ is a Banach space, the sequence $\left\{16^{n} f\left(2^{-n} x\right)\right\}$ converges. Now, we define $Q: X \rightarrow Y$ by $Q(x)=\lim _{n \rightarrow \infty} 16^{n} f\left(2^{-n} x\right)$, for all $x \in X$. The rest of proof is similar to that of Theorem 8 . 
Theorem 10. Let $\phi: X \times X \rightarrow Y$ be a function such that

$$
\sum_{i=0}^{\infty} \frac{\phi\left(0,2^{i} x\right)}{2^{i}}<\infty
$$

for all $x \in X$ with the condition

$$
\lim _{n \rightarrow \infty} \frac{\phi\left(2^{n} x, 2^{n} y\right)}{2^{n}}=0
$$

for all $x, y \in X$. Suppose that a function $f: X \rightarrow Y$ satisfies the inequality

$$
\left\|D_{f}(x, y)\right\| \leq \phi(x, y)
$$

for all $x, y \in X$, and $f(0)=0$. Then there exist a unique additive function $A: X \rightarrow Y$ and a unique quartic function $Q: X \rightarrow Y$ satisfying (1) and

$$
\begin{aligned}
\|f(x)-A(x)-Q(x)\| & \\
\leq \frac{1}{32} \sum_{i=0}^{\infty} & \left(\frac{1}{16^{i}}\left[\phi\left(0,2^{i} x\right)+\phi\left(0,-2^{i} x\right)\right]\right. \\
& \left.+\frac{32}{2^{i}}\left[\phi\left(0,2^{i} x\right)+\phi\left(0,-2^{i} x\right)\right]\right)
\end{aligned}
$$

for all $x \in X$.

Proof. We have

$$
\left\|D_{f_{\mathrm{e}}}(x, y)\right\| \leq \frac{1}{2}[\phi(x, y)+\phi(-x,-y)]
$$

for all $x, y \in X$. Since $f_{e}(0)=0$ and $f_{e}$ is an even function, then by Theorem 6 , there exists a unique additive function $A: X \rightarrow Y$ satisfying

$$
\left\|f_{e}(x)-A(x)\right\| \leq \frac{1}{2} \sum_{i=0}^{\infty} \frac{\phi\left(0,2^{i} x\right)+\phi\left(0,-2^{i} x\right)}{2 \times 2^{i}}
$$

for all $x \in X$. On the other hand $f_{0}$ is odd function and

$$
\left\|D_{f_{0}}(x, y)\right\| \leq \frac{1}{2}[\phi(x, y)+\phi(-x,-y)]
$$

for all $x, y \in X$. Then by Theorem 8 , there exists a unique quartic function $Q: X \rightarrow Y$ such that

$$
\left\|f_{o}(x)-Q(x)\right\| \leq \frac{1}{16} \sum_{i=0}^{\infty} \frac{\phi\left(0,2^{i} x\right)+\phi\left(0,-2^{i} x\right)}{2 \times 16^{i}}
$$

for all $x \in X$. Combining (55) and (56) to obtain (54). This completes the proof of theorem. 
Using Theorem 10, we solve the Hyers-Ulam stability problem for the functional equation (6) in the following corollary.

Corollary 11. Let $\epsilon$ be a positive real numbers, and let $f: X \rightarrow Y$ be a function satisfies

$$
\left\|D_{f}(x, y)\right\| \leq \epsilon
$$

for all $x, y \in X$. Then there exists a unique additive function $A: X \rightarrow Y$ and a unique quartic function $Q: X \rightarrow Y$ satisfying (6), and

$$
\|f(x)-A(x)-Q(x)\| \leq \frac{61}{15} \epsilon
$$

for all $x \in X$.

Proof. By considering $\phi(x, y)=\epsilon$, for all $x, y \in X$ in Theorem 10, we have

$$
\begin{aligned}
\|f(x)-A(x)-Q(x)\| & \leq \frac{1}{32} \sum_{i=0}^{\infty}\left(\frac{1}{16^{i}} 2 \epsilon+\frac{32}{2^{i}} 2 \epsilon\right) \\
& \leq \frac{2 \epsilon}{32} \sum_{i=0}^{\infty}\left(\frac{1}{16^{i}}+\frac{32}{2^{i}}\right) \\
& \leq \frac{\epsilon}{16}\left[\left(1-\frac{1}{16}\right)^{-1}+32\left(1-\frac{1}{2}\right)^{-1}\right] \\
& \leq \frac{\epsilon}{16}\left(\frac{16}{15}+64\right) \\
& \leq \frac{61}{15} \epsilon, \text { for all } x \in X .
\end{aligned}
$$

Corollary 12. Let $\theta \geq 0, p<1$. Suppose $f: X \rightarrow Y$ satisfies the inequality

$$
\left\|D_{f}(x, y)\right\| \leq \theta\left(\|x\|^{p}+\|y\|^{p}\right)
$$

for all $x, y \in X$ and $f(0)=0$. Then there exists a unique additive function $A: X \rightarrow Y$ and a unique quartic function $Q: X \rightarrow Y$ satisfying (6), and

$$
\|f(x)-A(x)-Q(x)\| \leq \frac{\theta}{16}\left(\frac{1}{1-2^{p-4}}+\frac{32}{1-2^{p-1}}\right)\|x\|^{p}
$$

for all $x \in X$. 
Proof. Taking $\phi(x, y)=\theta\left(\|x\|^{p}+\|y\|^{p}\right)$, for all $x, y \in X$ in Theorem 10, we arrive at

$$
\begin{aligned}
\|f(x)-A(x)-Q(x)\| & \leq \frac{1}{32} \sum_{i=0}^{\infty}\left(\frac{1}{16^{i}}\left[\phi\left(0,2^{i} x\right)+\phi\left(0,-2^{i} x\right)\right]\right. \\
& \left.+\frac{32}{2^{i}}\left[\phi\left(0,2^{i} x\right)+\phi\left(0,-2^{i} x\right)\right]\right) \\
& \leq \frac{1}{32} \sum_{i=0}^{\infty}\left(\frac{2 \theta}{16^{i}} 2^{p i}+\frac{32 \theta}{2^{i}} 2^{p i+1}\right)\|x\|^{p} \\
& \leq \frac{\theta}{16}\left(\frac{1}{1-2^{p-4}}+\frac{32}{1-2^{p-1}}\right)\|x\|^{p}
\end{aligned}
$$

for all $x \in X$.

\section{References}

[1] Abasalt Bodaghi, Stability of a Quartic Functional Equation, The Scientific World Journal, 2014, Art. ID 752146 (2014), 9 pages.

[2] T. Aoki, On the stability of the linear transformation in Banach spaces, J. Math. Soc. Japan, 2 (1950), 64-66.

[3] Charinthip Hengkrawit and Anurak Thanyacharoen, A general solution of a quartic functional equation and its stability, Int. J. Pure and Appl. Math., 85(4) (2013), 691-706.

[4] J.K. Chung and P.K. Sahoo, On the general solution of a quartic functional equation, Bull. Korean Math. Soc., 40(4) (2003), 565-576.

[5] M.E. Gordji, S. Abbaszadeh and C. Park, On the stability of a generalized quadratic and quartic type functional equation in quasi-Banach spaces, J. Inequal. Appl. 2009, Article ID 153084 (2009), 26 pages.

[6] M.E. Gordji, S.K. Gharetapeh, Ch. Park and S. Zolfaghri, Stability of an additive-cubic-quartic functional equation, Adv. in Difference Equations, 2009, Art. 395693 (2009), 20 pages.

[7] M.E. Gordji, S.K. Gharetapeh, Ch. Park and S. Zolfaghari, Stability of an Additive-Cubic-Quartic Functional Equation, Advances in Difference Equations, 2009, Art.ID 395693 (2009), 20 pages. 
[8] M.E. Gordji, Stability of a functional equation deriving from quartic and additive functions, Bull. Korean Math. Soc., 47(3) (2010), 491-502.

[9] Heejeong Koh and Dongseung Kang, Solution and Stability of EulerLagrange-Rassias Quartic Functional Equations in Various Quasi-normed Spaces, Abst. Appl. Anal., 2013, Art. ID 908168 (2013), 8 pages.

[10] D.H. Hyers, On the stability of the linear functional equation, Proc. Nat. Acad. Sci. U.S.A. 27 (1941), 222-224.

[11] S.M. Jung, On the Hyers-Ulam-Rassias stability of a quadratic functional equation, J. Math. Anal. Appl. 232 (1999), 384-393.

[12] J.R. Lee, D.Y. Shin and Ch. Park, Hyers-Ulam stability of functional equations in matrix normed spaes, J. Inequl. Appl., 22 (2013), 11 pages.

[13] S.H. Lee, S.M. Im and I.S. Hwang, Quartic functional equations, J. Math. Anal. Appl., 307 (2005), 387-394.

[14] M. Mohamadi, Y.J. Cho, C. Park, P. Vetro and R. Saadati, Random Stability of an Additive-Quadratic-Quartic Functional Equation, J. Inequ. Appl. 2010, Art.ID 754210 (2010), 18 pages.

[15] A. Najati, The generalized Hyers-Ulam-Rassias stability of a cubic functional equation, Turk. J. Math. 31 (2007), 395-408.

[16] Ch. Park, S.W. Jo and D.Y. Kho, On the stability of AQCQ-functional equation, J. Chungcheon Math. Soc., 22(4) (2009), 757-770.

[17] Ch. Park, Fuzzy stability of an Additive-Quadratic-Quartic functional equation, J. Inequ. Appl., 2010, Art. ID 253040 (2010), 22 pp.

[18] K. Ravi, J.M. Rassias, M. Arunkumar and R. Kodandan, Stability of a generalized mixed type additive, quadratic, cubic and quartic functional equation, J. Ineq. Pure \& Appl. Math. 10(4) (2009), 1-29.

[19] K. Ravi and B.V. Senthil Kumar, Ulam-Gavruta-Rassias stability of Rassias Reciprocal functional equation, Global J. of Appl. Math. and Math. Sci. 3(1-2) (Jan-Dec 2010), 57-79.

[20] K. Ravi, J.M. Rassias and B.V. Senthil Kumar, Ulam stability of Generalized Reciprocal Functional Equation in Several Variables, Int. J. App. Math. Stat. 19(D10) (2010), 1-19. 
[21] K. Ravi, J.M. Rassias and B.V. Senthil Kumar, Ulam stability of Reciprocal Difference and Adjoint Functional Equations, The Australian J. Math. Anal. Appl., 8(1), Art. 13 (2011), 1-18.

[22] Th.M. Rassias, On the stability of the linear mapping in Banach spaces, Proc. Amer. Math. Soc. 72 (1978), 297-300.

[23] S.M. Ulam, A collection of mathematical problems, Interscience Publishers, Inc.New York, (1960). 